\title{
Successful Sodium Level Correction with a 3\% Saline Bolus before Intermittent Hemodialysis for a Patient with Severe Hyponatremia Accompanied by Acute Kidney Injury
}

\author{
Miyuki Kodama, Daisuke Hirai, Seijiro Tsuji, Jaegi Shim, Mitsuteru Koizumi, \\ Koichi Seta and Kensei Yahata
}

\begin{abstract}
:
A 60-year-old man presented to the emergency department with lightheadedness. He had severe hyponatremia (109 mEq/L) complicated by acute kidney injury (AKI) (serum creatinine: $9.08 \mathrm{mg} / \mathrm{dL}$ ). Because he was somnolescent, his hyponatremia was initially treated by administering a $130-\mathrm{mL}$ bolus of $3 \%$ saline 2 to 5 times per day for 5 days. He subsequently underwent intermittent hemodialysis without any neurological problems. Previous reports have described patients with hyponatremia and AKI being treated with continuous renal replacement therapy. However, our strategy might be a feasible, low-cost treatment strategy of treating patients with hyponatremia and AKI who do not require immediate hemodialysis.
\end{abstract}

Key words: hyponatremia, acute kidney injury, $3 \%$ saline bolus administration, hemodialysis, osmotic demyelination syndrome

(Intern Med 60: 2645-2649, 2021)

(DOI: 10.2169/internalmedicine.6667-20)

\section{Introduction}

Hyponatremia is the most common disorder of body fluids and the electrolyte balance encountered in clinical practice. In serious cases involving disturbances of consciousness, it is important to treat hyponatremia quickly. However, it is necessary to adjust patients' sodium levels carefully in such cases to prevent osmotic demyelination syndrome (ODS).

We herein report a case of severe hyponatremia complicated with acute kidney injury (AKI). The patient's hyponatremia was initially treated by administering a bolus of hypertonic saline, and he subsequently underwent intermittent hemodialysis.

\section{Case Report}

A 60-year-old man presented to the emergency department with lightheadedness. His medical history included schizophrenia, for which he had been taking levomepro- mazine, biperiden, haloperidol, distigmine, flunitrazepam, promethazine, phenobarbital, and suvorexant. He had been drinking 48 glasses of water every day. He had noticed a decreased urinary volume three days before he came to the hospital. He developed lightheadedness and fell down several times the next day. His symptoms worsened, and he could not walk on the day of his admission. However, he might not have been able to accurately remember the abovementioned time sequence. There were no witnesses to these events because he lived alone.

His Glasgow coma scale was E3 V5M6, and he was somnolescent when he arrived at our hospital. He was $164.5 \mathrm{~cm}$ tall and weighed $79.2 \mathrm{~kg}$. His baseline body weight was not known. On an examination, his blood pressure was 135/78 $\mathrm{mmHg}$, and his pulse rate was 105 beats/minute. He displayed a tremor and bilateral pedal edema. An initial investigation revealed severe hyponatremia $(109 \mathrm{mEq} / \mathrm{L})$ complicated by a decreased renal function, a serum creatinine level of $9.08 \mathrm{mg} / \mathrm{dL}$, a blood urea nitrogen level of $63 \mathrm{mg} / \mathrm{dL}$, and rhabdomyolysis (creatine kinase: 22,615 IU/L) (Table). His baseline serum creatinine level was $1.0 \mathrm{mg} / \mathrm{dL}$, and his

Department of Nephrology, National Hospital Organization Kyoto Medical Center, Japan 
Table. Laboratory Data.

\begin{tabular}{|c|c|c|}
\hline & & Normal range \\
\hline Total protein $(\mathrm{g} / \mathrm{dL})$ & 5.1 & $6.3-7.9$ \\
\hline Serum albumin $(\mathrm{g} / \mathrm{dL})$ & 2.7 & $3.4-4.9$ \\
\hline Lactate dehydrogenase (IU/L) & 682 & $119-229$ \\
\hline Creatine kinase (IU/L) & 22,615 & $62-287$ \\
\hline Blood urea nitrogen $(\mathrm{mg} / \mathrm{dL})$ & 63 & $8-22$ \\
\hline Serum creatinine $(\mathrm{mg} / \mathrm{dL})$ & 9.08 & $0.55-1.14$ \\
\hline Serum sodium $(\mathrm{mEq} / \mathrm{L})$ & 109 & $138-146$ \\
\hline Serum potassium $(\mathrm{mEq} / \mathrm{L})$ & 3.8 & $3.6-4.9$ \\
\hline Serum chloride (mEq/L) & 78 & $99-109$ \\
\hline Aspartate transaminase (IU/L) & 333 & $13-33$ \\
\hline Alanine transaminase (IU/L) & 139 & $8-42$ \\
\hline Alkaline phosphatase (IU/L) & 333 & $115-359$ \\
\hline$\gamma$-Glutamyl transpeptidase (IU/L) & 46 & $10-47$ \\
\hline Total cholesterol (mg/dL) & 182 & $150-219$ \\
\hline High density lipoprotein cholesterol (mg/dL) & 58 & $40-80$ \\
\hline Low density lipoprotein cholesterol (mg/dL) & 90 & $70-139$ \\
\hline Triglycerides (mg/dL) & 88 & $50-149$ \\
\hline Serum glucose (mg/dL) & 115 & $69-104$ \\
\hline White blood cell count $\left(\times 10^{3} / \mu \mathrm{L}\right)$ & 11.9 & $3.5-8.5$ \\
\hline Red blood cell count $\left(\times 10^{6} / \mu \mathrm{L}\right)$ & 3.45 & $4.3-5.7$ \\
\hline Hemoglobin (g/dL) & 10.4 & $13.5-17$ \\
\hline Platelet $\left(\times 10^{3} / \mu \mathrm{L}\right)$ & 169 & $150-350$ \\
\hline Thyriod stimulating hormone $(\mu \mathrm{U} / \mathrm{mL})$ & 0.415 & $0.350-4.940$ \\
\hline Free T4 (ng/dL) & 0.78 & $0.70-1.48$ \\
\hline Adrenocorticotropic hormone (pg/mL) & 21.8 & $7.2-63.3$ \\
\hline Cortisol $(\mu \mathrm{g} / \mathrm{dL})$ & 15.18 & $7.07-19.6$ \\
\hline Proteinase 3 antineutrophil cytoplasmic antibody & $(-)$ & \\
\hline Myeloperoxidase antineutrophil cytoplasmic antibody & $(-)$ & \\
\hline Antiglomerular basement membrane antibody & $(-)$ & \\
\hline Antistreptolysin-O (IU/mL) & $<10$ & $0-200$ \\
\hline Urine urea nitrogen $(\mathrm{mg} / \mathrm{dL})$ & 85 & \\
\hline Urine creatinine (mg/dL) & 93.7 & \\
\hline Urinary sodium (mEq/L) & 79 & \\
\hline Urinary potassium $(\mathrm{mEq} / \mathrm{L})$ & 15 & \\
\hline Urinary chloride (mEq/L) & 57 & \\
\hline Urinary specific gravity & 1.01 & \\
\hline Urinary protein & $3+$ & \\
\hline Urinary glucose & $(-)$ & \\
\hline Urinary ketones & $(-)$ & \\
\hline Urinary occult blood & $3+$ & \\
\hline \multicolumn{3}{|l|}{ Urinary sediment } \\
\hline Red blood cell (/high power field) & $5-9$ & \\
\hline White blood cell (/high power field) & $>100$ & \\
\hline \multicolumn{3}{|l|}{ Arterial blood gas } \\
\hline $\mathrm{pH}$ & 7.367 & $7.350-7.450$ \\
\hline $\mathrm{pCO}_{2}(\mathrm{mmHg})$ & 33.9 & $32.0-48.0$ \\
\hline $\mathrm{HCO}_{3}^{-}(\mathrm{mmol} / \mathrm{L})$ & 19.0 & $21.0-27.0$ \\
\hline
\end{tabular}

serum sodium level had been $138 \mathrm{mEq} / \mathrm{L}$ one year earlier. His blood glucose and lipid levels were within normal limits, and his serum total protein level was below the normal limit (Table). These findings were consistent with hypotonic hyponatremia. The patient's urinary sodium, potassium, creatinine, and urea nitrogen levels were $79 \mathrm{mEq} / \mathrm{L}, 15 \mathrm{mEq} / \mathrm{L}$, $93.7 \mathrm{mg} / \mathrm{dL}$, and $85 \mathrm{mg} / \mathrm{dL}$, respectively (Table). His frac- tional excretion values for sodium and urea nitrogen were $7.02 \%$ and $27.8 \%$, respectively. Echography showed normalsized kidneys, and the diameter of the inferior vena cava was $16 \mathrm{~mm}$ and exhibited respiratory fluctuations. Chest Xray showed a cardiothoracic ratio of $55.5 \%$. Further investigations of the hyponatremia produced unremarkable findings, including a normal thyroid function and normal serum 


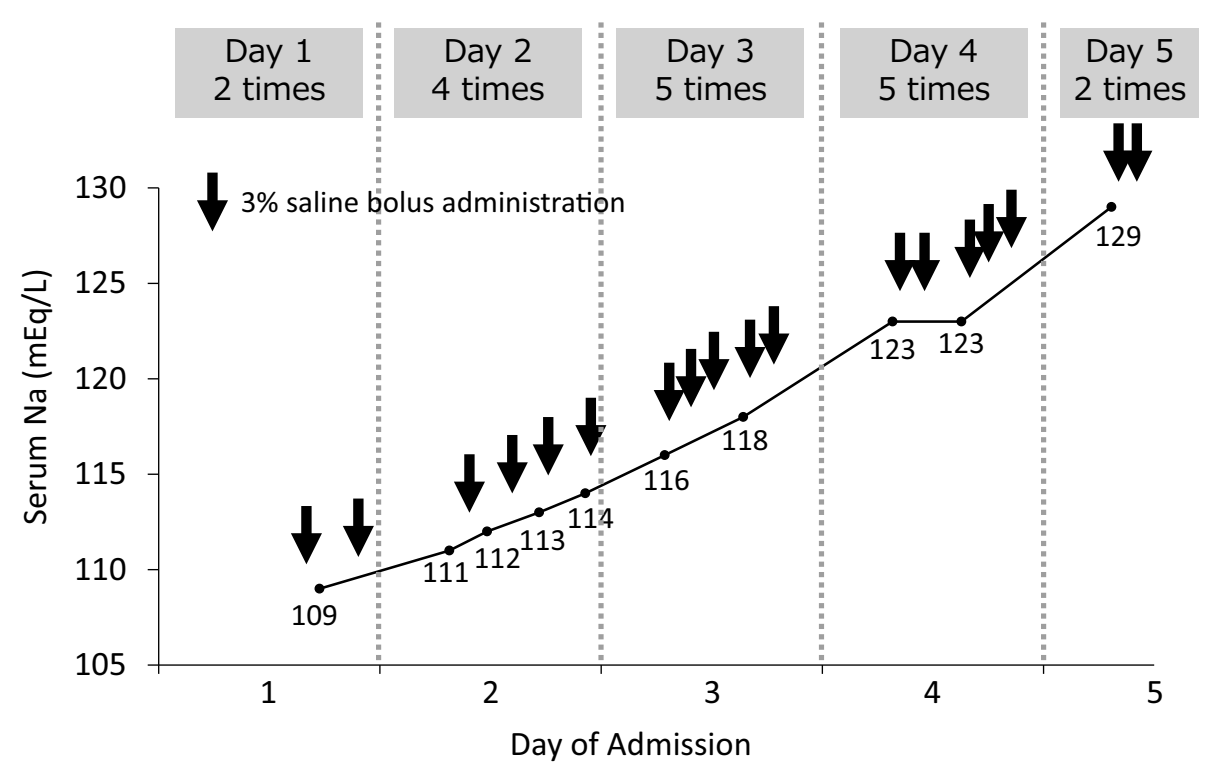

Figure 1. Changes in the serum sodium concentration seen during correction with $3 \%$ saline.

cortisol levels. Further investigations of the patient's renal dysfunction were also performed. Tests for proteinase-3 antineutrophil cytoplasmic antibody (ANCA), myeloperoxidase ANCA, and anti-glomerular basement membrane antibody were negative, and the level of antistreptolysin-O was within the normal range. The patient had not started taking any new medications in the past few months.

Water restriction and immediate treatment with hypertonic saline $(3 \% \mathrm{NaCl})$ for severe hyponatremia were initiated on the 1st hospital day. As hyperkalemia, acidemia, and pulmonary congestion were absent, immediate hemodialysis was considered unnecessary. We administered a $130-\mathrm{mL}$ bolus of $3 \%$ saline 2 to 5 times per day for 5 days while monitoring the patient's serum sodium level to ensure that it did not rise more than $10 \mathrm{mEq} / \mathrm{L} /$ day (Fig. 1). The patient's serum sodium level increased by $1-2 \mathrm{mEq} / \mathrm{L}$ after the administration of each bolus (Fig. 1). On the 6th hospital day, we stopped correcting the patient's serum sodium level because followup examinations indicated that his sodium level had reached $130 \mathrm{mEq} / \mathrm{L}$ (Fig. 1). Oliguria persisted during this period, and the patient's body weight rose $4 \mathrm{~kg}$ (Fig. 2). His serum creatinine level also rose to $16.9 \mathrm{mg} / \mathrm{dL}$, and intermittent hemodialysis was started on the 6th hospital day (Fig. 2). On the 13th hospital day, after 3 rounds of intermittent hemodialysis, the patient's serum creatinine level was 10.0 $\mathrm{mg} / \mathrm{dL}$, and his urinary volume was $1,199 \mathrm{~mL} /$ day (Fig. 2). He did not suffer any subsequent recurrence of the renal dysfunction despite not undergoing further hemodialysis. On the 22nd hospital day, his serum sodium level was 137 $\mathrm{mEq} / \mathrm{L}$, his serum creatinine level was $2.94 \mathrm{mg} / \mathrm{dL}$, and his body weight was $70.4 \mathrm{~kg}$ (Fig. 2). He was able to restrict his water intake during his hospitalization, and he was discharged on the 23rd hospital day.

\section{Discussion}

Hyponatremia is one of the most common water balance disorders and is often difficult to diagnose. Two sets of treatment guidelines for hyponatremia have been developed by professional organizations, one from the United States (1) and the other from Europe (2). Initial differentiation between hypotonic and non-hypotonic hyponatremia is recommended because these conditions require different management strategies. We first excluded non-hypotonic hyponatremia, as the patient's laboratory data showed that his blood glucose and lipid levels were within normal limits, and his serum total protein level was below the normal limit.

Hypotonic hyponatremia is usually divided into hypovolemic, euvolemic, and hypervolemic hyponatremia. The United States guideline also employs this approach (1). Based on his examination findings, it was considered that our patient was suffering from euvolemic or hypervolemic hyponatremia. Further examinations did not find any obvious causes of the hyponatremia, such as hypothyroidism or decreased cortisol secretion. The European guideline committee proposed an algorithm that prioritizes urinary osmolality $\left(\mathrm{U}_{\mathrm{Osm}}\right)$ and the urinary sodium level $\left(\mathrm{U}_{\mathrm{Na}}\right)$ over volume status (2). However, we considered that it would have been difficult to use this algorithm in this case because the patient had renal dysfunction and anuria. The patient had been taking levomepromazine and haloperidol, which can cause hyponatremia as an adverse effect. Although he had been taking these medications for many years, they might have caused his condition.

We also considered the patient's pathophysiology. The hyponatremia seen in this case was considered to have been caused by polydipsia, AKI, and medications. Regarding the AKI, examinations suggested that the patient had intrinsic 


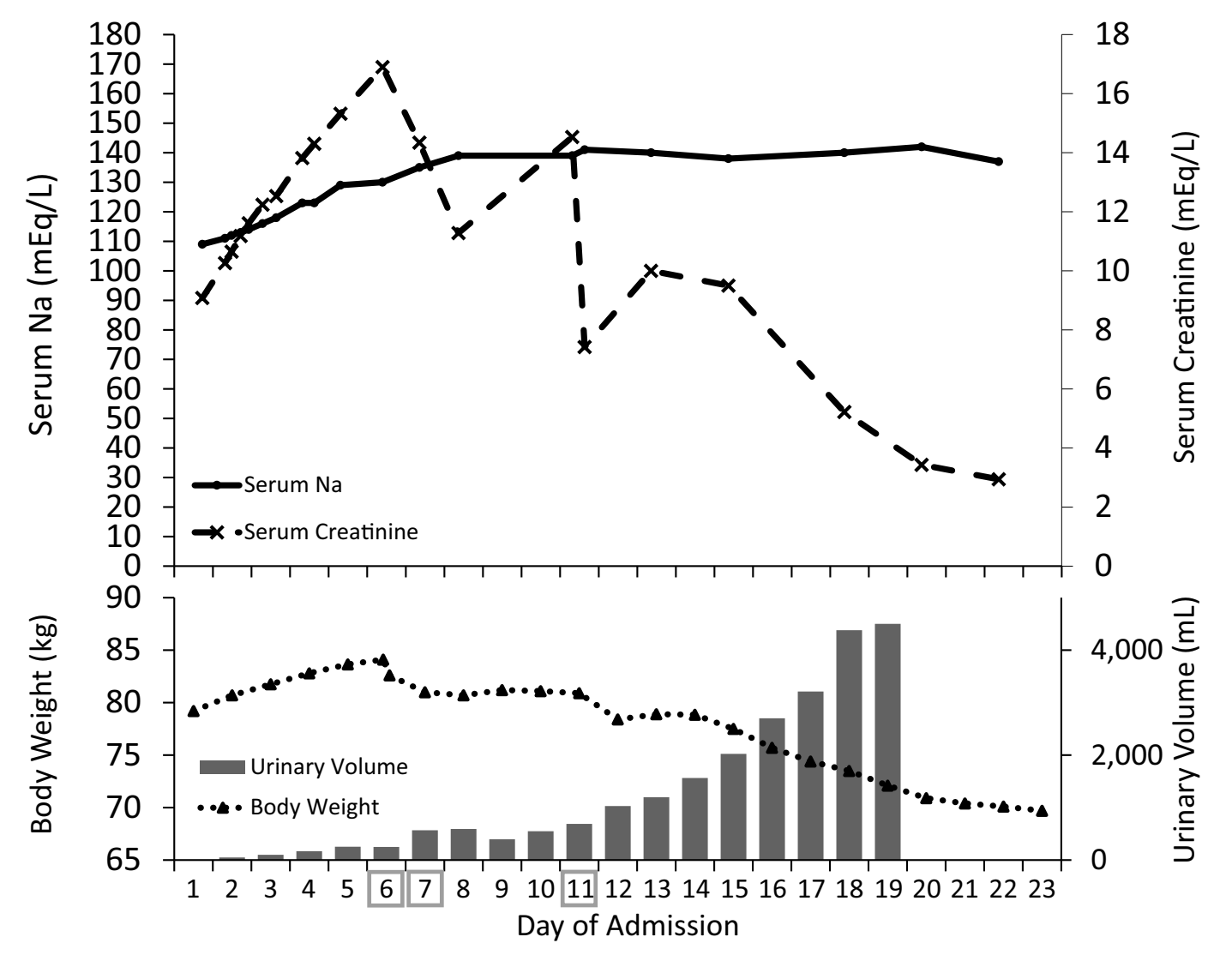

Figure 2. Clinical course. Intermittent hemodialysis was conducted on the days of the number surrounded with a square.

renal failure caused by rhabdomyolysis. There were two possibilities regarding the pathophysiology of the patient's condition, (i) the AKI preceded the hyponatremia, and (ii) the hyponatremia preceded the AKI. These situations might have played out as follows: (i) the patient suffered a fall first and then developed rhabdomyolysis, which resulted in AKI; he still continued drinking a lot of water, even though he could not excrete enough water as urine; thus, he developed hyponatremia; alternatively, (ii) the patient developed hyponatremia first because of polydipsia and/or medications, and then he suffered a fall as one of the symptoms of the hyponatremia; he subsequently developed rhabdomyolysis, which resulted in AKI. He might not have remembered the time series of these events accurately, and there were no witnesses; therefore, the actual cause of the patient's pathophysiology remains unknown.

In recent years, the treatment for acute hyponatremia has switched toward the administration of hypertonic saline as a fixed bolus. The two sets of guidelines mentioned above outline the current approach to hyponatremia $(1,2)$. Both sets of guidelines recommend the administration of hypertonic saline $(3 \% \mathrm{NaCl})$ for acute or symptomatic hyponatremia. The United States guideline recommends the administration of a $100-\mathrm{mL}$ bolus of $3 \%$ saline over 10 minutes 3 times per day, as needed (1), and the European guideline recommends the administration of a $150-\mathrm{mL}$ bolus of $3 \%$ saline over 20 minutes 2-3 times per day, as needed, for hyponatremia with severe symptoms (2). The administration of a fixed bolus has some appealing aspects. First, especially in patients with cerebral edema, it is desirable to achieve a rapid partial correction of the serum sodium concentration. Second, it is not necessary to perform calculations in patients with acute problems when administering a fixed bolus, which eliminates the risk of calculation errors. Third, bolus therapy limits the risk of overcorrection, which commonly occurs after the continuous infusion of hypertonic saline. The two sets of guidelines agree that the maximum amount of saline administered should be around $10 \mathrm{mEq} / \mathrm{L}$ per day for both acute and chronic hyponatremia, as an acute increase in extracellular tonicity can cause $\operatorname{ODS}(1,2)$.

In the present case, we decided to start immediate treatment with hypertonic saline because the patient had a consciousness disorder caused by hyponatremia. Furthermore, it seemed that his hyponatremia would not resolve without active intervention due to his renal dysfunction. As hyperkalemia, acidemia, and pulmonary congestion were absent, immediate hemodialysis was not necessary. However, it was also considered that he would soon need to undergo hemodialysis because of anuria. Therefore, we had to consider whether he could receive hemodialysis, which might have increased his serum sodium level rapidly, even if he still had hyponatremia.

Elevated blood urea levels are considered to protect uremic patients from ODS when hyponatremia is rapidly corrected using hemodialysis. A case report described a patient with acute-on-chronic renal failure and hyponatremia who 
tolerated hemodialysis well without any neurological effects despite a serum sodium level correction rate of $3 \mathrm{mEq} / \mathrm{L} /$ h (3). A previous study indicated that high brain levels of urea might help protect against the dehydrating effects of rapid sodium correction in azotemic rats with hyponatremia (4). However, the protective effect of elevated blood urea nitrogen levels might be limited, and a case in which uremic patient with severe hyponatremia that was treated with hemodialysis presented with ODS has been reported (5). Therefore, we prioritized treating the patient's hyponatremia in this case.

Some case reports have described cases in which patients with hyponatremia and AKI were treated with continuous renal replacement therapy (CRRT) by modifying the sodium concentration of the dialysate and replacement fluid based on the serum sodium concentration $(6,7)$. They suggested that CRRT has advantages in terms of its ability to correct plasma sodium values safely. However, adding or exchanging a commercially prepared fluid can be complicated due to the sodium concentration calculations required, which can lead to mistakes. These issues are further compounded by the high cost of CRRT.

In the present case, we administered a $130-\mathrm{mL}$ bolus of $3 \%$ saline for 5 days while monitoring the patient's serum sodium level to ensure that it did not increase by more than $10 \mathrm{mEq} / \mathrm{day}$, as stated in the sets of guidelines mentioned above. As the patient had anuria, we monitored his serum sodium level carefully and managed the speed at which saline was administered appropriately to avoid overcorrection. When he came to the hospital, we determined that he was either not experiencing extracellular fluid (ECF) overload or was only experiencing slight ECF overload, as he only exhibited bilateral pedal edema. Echography showed that the diameter of the inferior vena cava was normal, and chest $\mathrm{X}$ ray did not show an increased cardiothoracic ratio. It was considered that his total body fluid level was probably excessive because severe hyponatremia can cause increases in intracellular fluid (ICF) levels. These findings were consistent with our views about the patient's pathophysiology, i.e. that the hyponatremia was caused by polydipsia, AKI, and medications. It was predicted that the administration of saline would increase the ECF volume. We monitored the patient's body weight and respiratory status carefully. In addition, we made the necessary preparations to allow us to per- form hemodialysis if he showed any signs of respiratory dysfunction, such as decreased oxygenation. We consider that we succeeded in treating both severe hyponatremia and AKI by administering hypertonic saline as a fixed bolus before intermittent hemodialysis. This treatment might reduce the risk of ODS and does not require any modification of the dialysate or calculation of the sodium concentration required for hemodialysis, although careful management is needed.

Administering hypertonic saline as a fixed bolus before intermittent hemodialysis might be a feasible, low-cost treatment strategy for patients with severe hyponatremia accompanied by AKI who do not require immediate hemodialysis.

Informed consent was obtained from the patient in this study.

The authors state that they have no Conflict of Interest (COI).

\section{References}

1. Verbalis JG, Goldsmith SR, Greenberg A, et al. Diagnosis, evaluation, and treatment of hyponatremia: expert panel recommendations. Am J Med 126: S1-S42, 2013.

2. Spasovski G, Vanholder R, Allolio B, et al. Clinical practice guideline on diagnosis and treatment of hyponatraemia. Eur J Endocrinol 170: G1-G47, 2014.

3. Oo TN, Smith CL, Swan SK. Does uremia protect against the demyelination associated with correction of hyponatremia during hemodialysis? A case report and literature review. Semin Dial 16: 68-71, 2003.

4. Soupart A, Penninckx R, Stenuit A, Decaux G. Azotemia (48 h) decreases the risk of brain damage in rats after correction of chronic hyponatremia. Brain Res 852: 167-172, 2000.

5. Huang WY, Weng WC, Peng TI, Ro LS, Yang CW, Chen KH. Central pontine and extrapontine myelinolysis after rapid correction of hyponatremia by hemodialysis in a uremic patient. Ren Fail 29: 635-638, 2007.

6. Dangoisse C, Dickie H, Tovey L, Ostermann M. Correction of hyper- and hyponatraemia during continuous renal replacement therapy. Nephron Clin Pract 128: 394-398, 2014.

7. Yessayan L, Yee J, Frinak S, Szamosfalvi B. Treatment of severe hyponatremia in patients with kidney failure: role of continuous venovenous hemofiltration with low-sodium replacement fluid. Am J Kidney Dis 64: 305-310, 2014.

The Internal Medicine is an Open Access journal distributed under the Creative Commons Attribution-NonCommercial-NoDerivatives 4.0 International License. To view the details of this license, please visit (https://creativecommons.org/licenses/ by-nc-nd/4.0/).

\section{(C) 2021 The Japanese Society of Internal Medicine} Intern Med 60: 2645-2649, 2021 